\title{
Minimizing Spatial Deformation Method for Online Signature Matching
}

\author{
Bin $\mathrm{Li}^{1}$, Kuanquan Wang ${ }^{1}$, and David Zhang ${ }^{2}$ \\ ${ }^{1}$ Department of Computer Science and Technology, Harbin Institute of Technology \\ Harbin, China \\ \{1 bn, wangkq\} @hit.edu.cn \\ ${ }^{2}$ Department of Computing, The Hong Kong Polytechnic University \\ Kowloon, Hong Kong, SAR \\ csdzhang@ comp.polyu.edu.hk
}

\begin{abstract}
Elastic matching is an important algorithm for online signature verification. Most of elastic matching algorithms are based on dynamic time warping(DTW). In this paper, we propose a new points matching algorithm for online signature verification. After resampling a signature with equidistant spacing, we describe the resampling point by its $(3+1)$ demesional $((3+1) \mathrm{D})$ information: 3D spatial information (x-axis, $y$-axis, time) and a feature vector. A soft-assign matrix and two special restrictions are introduced for recording the correspondence between two signatures. With a deterministic annealing framework, the mapping between two sets of $(3+1) \mathrm{D}$ signature points is achieved by minimizing the warping energy of the thin-plate spline(TPS). Experimental results demonstrate that the proposed algorithm outperforms the DTW-based matching algorithms.
\end{abstract}

Keywords: Signature verification, $(3+1) \mathrm{D}$ information, warping energy, thinplate spline, deterministic annealing.

\section{Introduction}

The methods of online signature verification can be generally classified into two categories: function-based and parameter-based. Elastic matching algorithm is widely applied to parameter-based online signature verification, since the number of points(or strokes) is not consistent between any two signatures. For the past two decades, Dynamic time warping(DTW) has become a major algorithm for online signature verification. For example, A. Jain presented a DTW-based string matching algorithm which gives a penalty for the matching of two point sets with large differences in their number[1]. H. Feng proposed an alterant DTW algorithm named as extreme points warping(EPW) for online signature verification [2]. By accumulating the local distances of points, DTW is used to find the best matching path, in terms of the least global cost, between an input signal and a template. However, the spatial positional relation of the signature point is not considered in DTW-based elastic matching algorithm. Actually, this relation is very important for matching and verifying. For the correspondence of two pure 2D (or 3D) spatial point 
sets, H. Chui presented a point matching algorithm based on soft-assign and TPS[3]. It is also not satisfied to online signature points matching, since local features of the signature point are not considered.

In this paper, considering the spatial information and local features of a signature point, we describe a signature point by its $(3+1) \mathrm{D}$ information: $3 \mathrm{D}$ spatial information (x-axis, $\mathrm{y}$-axis, time) and a feature vector. Based on minimizing the warping energy(WE) of thin-plate spline(TPS) mapping, an elastic matching algorithm is proposed for the matching of two $(3+1) \mathrm{D}$ signature point sets.

\section{$2(3+1) D$ Description of the Signature Point}

With a fixed sampling frequency, a signature can be captured and described by a series of points $\left\{\left(x_{i}, y_{i}, t_{i}\right), i=1,2, \ldots, M\right\}$, where $x_{i}, y_{i}$ and $t_{i}$ is the $\mathrm{x}-, \mathrm{y}$ - and timecoordinates of $i$ th signature point. In order to evaluate our algorithm with DTW, we use the same preprocessing and feature extraction with Jain's [1]. After smoothing and normalizing, we resample the signature with equidistant spacing in $\mathrm{x}-\mathrm{y}$ plane. The original time information is directly kept down for each resampling point. The resampling signature can be defined as a point set

$$
\left\{p_{i}=\left(x_{i}, y_{i}, t_{i}\right), i=1, \ldots, N\right\},
$$

where $p_{i}$ is a resampling point and $N$ is the number of resampling points.

Jain evaluated 11 local features in his experiments and achieved the best result when selecting the feature set consisting of the features $\delta x, \delta y, \sin \alpha, \cos \alpha$, and the absolute speed $v$ between two resampling points. The feature computation is described in Fig. 1. In this paper, we construct a feature vector for each signature point by these 5 local features. The feature vector is named as a feature dimension opposite to $3 \mathrm{D}$ spatial information of the point.

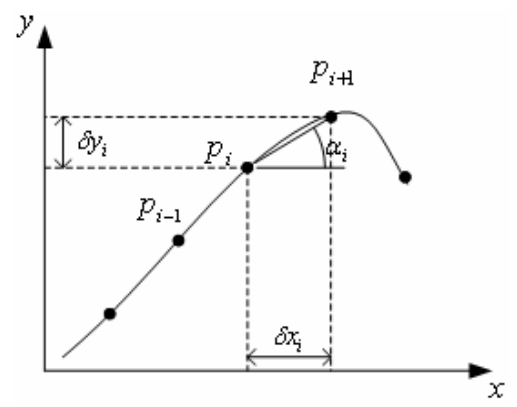

Fig. 1. Feature description of a resampling point

By combining the 3D spatial information and the feature vector, a signature $S$ can be defined as: 


$$
S=\left\{\left(\left(x_{i}, y_{i}, t_{i}\right), F_{i}\right), i=1, \ldots, N\right\}, F_{i}=\left(\delta x_{i}, \delta y_{i}, \sin \alpha_{i}, \cos \alpha_{i}, v_{i}\right),
$$

where $F_{i}$ is a feature vector, and $N$ is the number of resampling points.

\section{Description of Elastic Matching Algorithm}

Assume we have two point sets $S^{a}$ and $S^{b}$ with known correspondence, which include 3D spatial homogeneous coordinates sets $P^{a}$ and $P^{b}$, and feature vector sets $F^{a}$ and $F^{b}$. The 3D spatial TPS mapping function $f: P^{b} \rightarrow P^{a}$ can be defined as following:

$$
f\left(P^{b}\right)=P^{b} \cdot d+U \cdot c
$$

where $d$ is a $4 \times 4$ affine transformation matrix and $c$ is a $N \times 4$ non-affine coefficient matrix, $U$ is the $N \times N$ 3D TPS kernel matrix consisting of $u_{i j}=-\left\|p_{i}^{b}-p_{j}^{b}\right\|$.

Considering the distance of feature vector sets $F^{a}$ and $F^{b}$, we can obtain the TPS mapping function $f$ by minimizing the following warping energy function:

$$
E_{T P S}(d, c)=\left\|P^{a}-P^{b} d-U c\right\|^{2}+\lambda_{1}\left\|F^{a}-F^{b}\right\|^{2}+\lambda_{2} \operatorname{trace}\left(c^{T} U c\right) .
$$

The QR decomposition and the least-squares solution are used to solve parameters $d$ and $c$ [4]. With the QR decomposition, we decompose $P^{b}$ into $N \times 4$ orthonormal matrix $Q_{1}, N \times(N-4)$ orthonormal matrix $Q_{2}$ and $4 \times 4$ upper triangular $R$. With the permutation of $c=Q_{2} \gamma$, the Eq.4 can be transformed to

$$
\begin{gathered}
E_{T P S}(d, \gamma)=\left\|Q_{2}^{T} P^{a}-Q_{2}^{T} U Q_{2} \gamma\right\|^{2}+\left\|Q_{1}^{T} P^{a}-R d-Q_{1}^{T} U Q_{2} \gamma\right\|^{2} \\
+\lambda_{1}\left\|F^{a}-F^{b}\right\|^{2}+\lambda_{2} \gamma^{T} Q_{2}^{T} U Q_{2} \gamma .
\end{gathered}
$$

By differentiating Eq. 5 w.r.t. $\gamma$ and $d$ and setting them to zeros, $d$ and $c$ can be calculated by:

$$
d=R^{-1} Q_{1}^{T}\left(P^{a}-U Q_{2} r\right), \quad c=Q_{2}\left(Q_{2}^{T} U Q_{2}+\lambda_{2} I\right)^{-1} Q_{2}^{T} P^{a} .
$$

Actually, the reason we introduce an elastic matching algorithm for online signature verification is that the correspondence of two signature point sets is unknown. Now we explain our elastic matching algorithm as follows.

Set $S^{a}$ and $S^{b}$ are two point sets with unknown correspondence whose point numbers are $K$ and $N$ respectively. A $K \times N$ soft-assign matrix $M$ consisting of $m_{i j} \in[0,1]$ is introduced to record the correspondence between $S^{a}$ and $S^{b}$. Intuitively, the larger $m_{i j}$ is, the bigger the possibility of point $s_{i}^{a}$ corresponding to 
$s_{j}^{b}$ is. An extra row and column are given to ensure that the accumulation of each row or column holds to 1 , i.e. $\sum_{i=1}^{K+1} m_{i j}=1, \forall \quad j \in\{1, \ldots, N\}, \sum_{j=1}^{N+1} m_{i j}=1, \forall i \in\{1, \ldots, K\}$.

For the feature vector, we define a distance matrix $D^{F}$ consisting of $d_{i j}=\left\|F_{i}^{a}-F_{j}^{b}\right\|$, where $\|\bullet\|$ is the Euclidean distance, to record feature distances between each pair of points in $S^{a}$ and $S^{b}$.

With the definitions of soft-assign matrix $M$ and distance matrix $D^{F}$, the TPS warping energy function (Eq.5) is redefined by following:

$$
\begin{gathered}
E_{T P S}(M, d, c)=\sum_{i=1}^{K} \sum_{j=1}^{N} m_{i j}\left(\left\|P_{i}^{a}-P_{j}^{b} d-U\left(P_{j}^{b}\right) c\right\|^{2}+\lambda_{1} d_{i j}{ }^{2}\right) \\
+\lambda_{2} \operatorname{trace}\left(c^{T} U c\right)+T \sum_{i=1}^{K} \sum_{j=1}^{N} m_{i j} \log m_{i j} .
\end{gathered}
$$

The first term and the second term are related to the energy of affine transformation and non-affine warping respectively. The third term is a entropy barrier function that ensures the positivity of matrix $M$. In order to make the soft-assign matrix $M$ evolving to a binary-assign matrix which will include the final best matching path, a determinate annealing framework is introduced. With a annealing rate $R^{\text {anneal }}$, the temperature parameter $T$, the controlling parameters $\lambda_{1}$ and $\lambda_{2}$ will be gradually reduced by $T=T^{\text {init }} R^{\text {anneal }}, \lambda_{1}=\lambda_{1}^{\text {init }} R^{\text {anneal }}$ and $\lambda_{2}=\lambda_{2}^{\text {init }} R^{\text {anneal }}$.

The algorithm is described as follows:

$\underline{\text { Step } 1}$ Initialize parameters $M, d, c, \lambda_{1}^{\text {init }}, \lambda_{2}^{\text {init }}$ and $T^{\text {init }}$.

$\underline{\text { Step } 2}$ Fixing the TPS mapping parameters $d$ and $c$, the equation $\partial E_{T P S} / \partial M=0$ is solved to achieve a new soft-assign matrix whose element can be calculated by

$$
m_{i j}^{\prime}=\exp \left(-\left(\sum_{i=1}^{K} \sum_{j=1}^{N} m_{i j}\left(\left\|P_{i}^{a}-P_{j}^{b} d-U\left(P_{j}^{b}\right) c\right\|^{2}+\lambda_{1} d_{i j}^{2}\right)\right) / T\right) .
$$

We normalize $m_{i j}^{\prime}$ across all rows and all columns, and update the soft-assign matrix $M$.

Step 3 With the updated soft-assign matrix $M$, we calculate the 3D spatial coordinates of each corresponding point by $P_{i}^{a} \leftarrow \sum_{i=1}^{K} m_{i j} P_{i}^{a}$ and then recalculate $d$ and $c$ by Eq.6. 
Step 4 Decrease $T, \lambda_{1}$ and $\lambda_{2}$, repeat step 2 and step 3 alternately until the algorithm converges. In our algorithm, the terminational condition of the convergence is $T \leq T_{\text {final }}$.

Step 5 The soft-assign matrix $M$ is regressed to a binary-assign matrix. When the algorithm has converged, we search each row and column of the soft-assign matrix $M$ for the maximum element, mark this element as 1 and the others as 0 . If the maximum is the $i$ th element of the extra row or column, that means the point corresponding to the $i$ th column or row is a mismatching point and will be eliminated.

In particular, there are two restrictions for the regression of soft-assign matrix $M$ to ensure the strict monotonicity and one-to-one mapping.

(1) Strict monotonicity:

$$
m_{i j}=0, \quad \forall\left\{\begin{array}{c}
1 \leq i \leq i_{0}-1, j_{0}+1 \leq j \leq N, \\
\text { or } \quad i_{0}+1 \leq i \leq K, 1 \leq j \leq j_{0}-1
\end{array},\right.
$$

where $m_{i_{0} j_{0}}=1, \quad i_{0} \in\{1, \ldots, K\}, j_{0} \in\{1, \ldots, N\}$.

(2) One-to-one mapping:

If both $m_{i j_{1}}$ and $m_{i j_{2}}$ which belong to $i$ th row of $M$ are equal to the maximum of $i$ th row, then

$$
\left\{\begin{array}{l}
m_{i j_{1}}=1, m_{i j_{2}}=0, \text { if } d_{i j_{1}} \leq d_{i j_{2}} \\
m_{i j_{1}}=0, m_{i j_{2}}=1, \text { else }
\end{array}\right.
$$

where $d_{i j_{1}}, d_{i j_{2}} \in D^{F}, i \in\{1, \ldots, K\}, j_{1}, j_{2} \in\{1, \ldots, N\}$.

The restriction for each column is the same.

Fig. 2 shows the elastic matching results that a template signature is compared with a genuine signature and a forgery.

\section{Experimental Results}

A digital tablet with $100 \mathrm{~Hz}$ sampling frequency was used for capturing the signatures. Over a period of 4 months, 102 Chinese volunteers took part in the data acquisition. Each writer was asked to write his/her signature 10 times. 1020 genuine signatures are collected in our database. About the forgery, 6 forgers were asked to imitate 5 signatures for each genuine signer, after they observed both the shape and the dynamic writing process of genuine signatures. Total 1530 signatures in our experimental database are used to evaluate our algorithm.

In our experiments, the annealing rate is set to 0.6 and parameters $\lambda_{1}^{\text {init }}$ and $\lambda_{2}^{\text {init }}$ are set to 1 . The initial value of $T$ is set to a value which is isometric with the largest square distance, which also contains the maximum of feature distance matrix $D^{F}$, for 

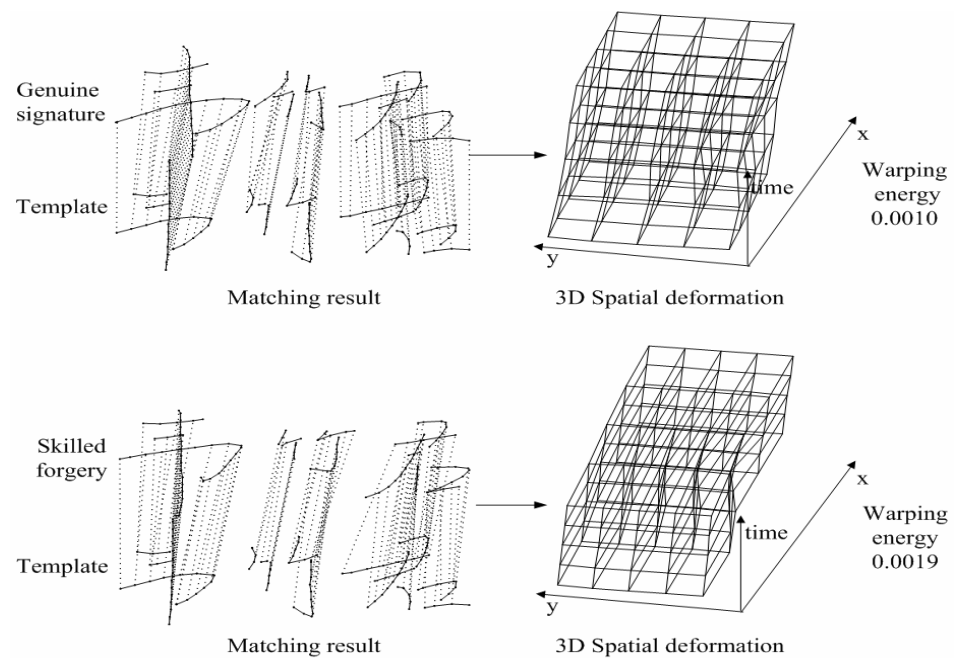

Fig. 2. Flexible matching based on TPS mapping

all possible pairs of points. The soft-assign matrix $M$ is initialized to a $K \times N$ matrix in which each element is set to $1 / \arg \max (K, N)$, and the outlier row and column are set to $1 /(100 \times \arg \max (K, N))$. $d$ and $c$ is initialized to a unit matrix and a zero matrix respectively. After about 7 8 times iteration, the proposed algorithm can converge at a good matching results.

In order to evaluate the proposed algorithm, with same database set, Jain's algorithm is implemented [1]. Based on the same preprocessing and feature extraction, Jain used DTW for elastic matching. For each signer, 3 and 5 genuine signatures are used in training, 5 samples of the rest genuine signatures and 5 skilled forgeries are used for testing. Table 1 gives the experimental comparison between Jain's DTW-based algorithm and the proposed algorithm. From Table 1, we can see that the verification performance using only the warping energy(WE) is already a little better than Jain's DTW-based algorithm. Combining WE and the Euclidean distance of local features(ED-F), the $(3+1) \mathrm{D}$ algorithm can achieve better results than Jain's. The best result of our algorithm yields an equal error rate of $1.9 \%$ for 5 references. The error tradeoff curves for the comparison are shown in Figure 3.

Table 1. Comparison between Jain's and the proposed algorithm for 3 and 5 references

\begin{tabular}{ccccc}
\hline $\begin{array}{c}\text { Reference } \\
\text { number }\end{array}$ & $\begin{array}{c}\text { Jain's DTW- } \\
\text { based }\end{array}$ & $(3+1) \mathrm{D}: \mathrm{WE}$ & $(3+1) \mathrm{D}: \mathrm{ED}-\mathrm{F}$ & $(3+1) \mathrm{D}$ \\
\hline 3 & $\mathrm{EER}=5.3 \%$ & $\mathrm{EER}=4.3 \%$ & $\mathrm{EER}=8.3 \%$ & $\mathrm{EER}=3.4 \%$ \\
5 & $\mathrm{EER}=3 \%$ & $\mathrm{EER}=2.8 \%$ & $\mathrm{EER}=5.8 \%$ & EER=1.9\% \\
\hline
\end{tabular}




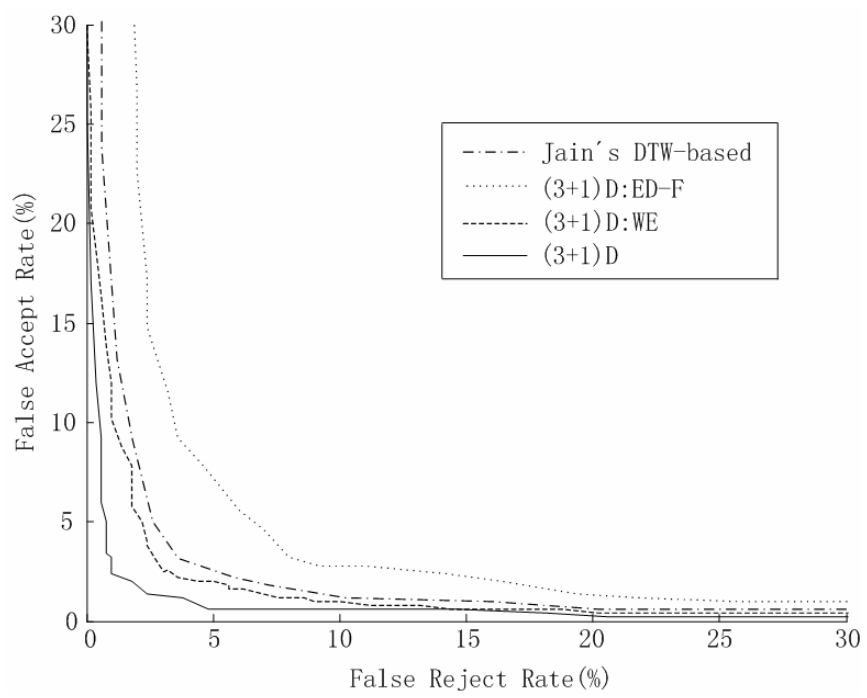

Fig. 3. Error tradeoff curves for the comparison between Jain's and the proposed algorithm

\section{References}

1. Jain, A.K., Griess, F.D., Connell, S.D.: Online Signature Verification. Pattern Recognition 35(2), 2963-2972 (2002)

2. Feng, H., Wah, C.C.: Online Signature Verification Using a New Extreme Points Warping Technique. Pattern Recognition Letters 24(16), 2943-2951 (2003)

3. Chui, H., Rangarajan, A.: A New Algorithm for Non-rigid Point Matching. In: IEEE Conf. on Computer Vision and Pattern Recognition, pp. 2044-2051. IEEE Computer Society Press, Los Alamitos (2000)

4. Wahba, G.: Spline Models for Observational Data. Society for Industrial and Applied Mathematics, PA (1990) 